\title{
DOSSIÊ
}

Sociologias, Porto Alegre, ano 16, no 37, set/dez 2014, p. 42-70

\section{Sustentabilidade entre interdisciplinaridade e propositividade: um olhar para artigos científicos em ecologia, economia, sociologia e antropologia}

\author{
ROBERTO DONATO DA SILUA JUNIOR \\ LEILA DA COSTA FERBEIRA* \\ THOMAS MICHAEL LEWINSOHN
}

\section{Resumo}

O objetivo deste artigo é oferecer uma análise da produção científica sobre sustentabilidade em conjuntos de artigos das áreas de ecologia, economia, sociologia e antropologia, a partir de quinze dentre os vinte e cinco artigos mais citados no sítio ISI -Web of Science em cada ciência, entre 1990 e 2010. Observaram-se os arranjos conceituais que configuram o termo nessas ciências, para compreender como elas abordam os hibridismos - as interfaces "humanos/não humanos" e "ciência/política"- presentes nos discursos sobre sustentabilidade. A análise concentrou-se na tensão entre disciplinaridade e interdisciplinaridade no contexto de estabelecimento da relação entre artefato científico e propositividade política. Para tanto, a leitura orientou-se por três dimensões da discursividade presentes nos artigos: as concepções de interação entre humanos e não humanos; as definições de risco e ameaça; e as estratégias de enfrentamento das questões de sustentabilidade. Essa tríade fundamentou-se na teoria da modernização reflexiva e nos estudos sociais da ciência. Observaram-se, nos quatro conjuntos, dois campos de possibilidades de articulação interdisciplinar denominados "ecologização" e "politização", ambos orientados por um sentido de "interdisciplinaridade por continuidade".

Palavras-chave: Interdisciplinaridade. Antropologia. Ecologia. Economia. Sociologia. Sustentabilidade.

\footnotetext{
Universidade Estadual de Campinas - UNICAMP, SP, Brasil.

** Universidade Estadual de Campinas - UNICAMP, SP, Brasil

*** Universidade Estadual de Campinas - UNICAMP, SP, Brasil
} 
Sustainability, between interdisciplinarity and proactiveness: an analysis of articles in ecology, economy, sociology and anthropology

\section{Abstract}

This article aims to provide an analysis of scientific literature on sustainability as expressed in sets of papers in the areas of ecology, economics, sociology and anthropology. Fifteen works were selected from the twenty-five most cited articles in each of these areas in the ISI-Web of Science, between 1990 and 2010. The conceptual frameworks that establish the term 'sustainability' in each area were observed, aiming to understand how they approach the dualisms - the interfaces "human / non-human" and "science / policy" - present in the discourses on sustainability. The analysis focused on the tension between disciplinarity and interdisciplinarity in establishing the relationship between scientific artifact and political proactiveness. To this end, the analysis was based on three dimensions of the discourse: the concepts of interaction between human and nonhuman; the definitions of risk and threat; and the strategies for coping with sustainability issues. This triad was based on the theory of reflexive modernization and on the social studies of science. In the four sets of papers, two possible fields for interdisciplinary connection were observed, which were called "ecologization" and "politicization", both guided by a sense of "interdisciplinarity by continuity".

Keywords: Interdisciplinarity. Anthropology. Ecology. Economy. Sociology. Sustainability.

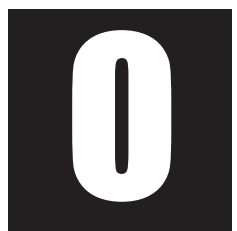

objetivo deste artigo é oferecer um olhar para diferentes perspectivas de sustentabilidade, por meio da análise dos artigos "mais citados" no sitio ISI-Web of Science nas áreas de ecologia, economia, sociologia e antropologia. A partir da leitura de um conjunto de quinze textos para cada campo abordado e de um recorte temporal que abrange o período entre 1990 e 2010, pretende-se observar como esses conjuntos tratam o tema, como forma de reconhecer os diferentes hibridismos - concebidos 
Sociologias, Porto Alegre, ano 16, no 37, set/dez 2014, p. 42-70

aqui como as interfaces "humanos/não humanos" e "ciência/política"presentes nesses discursos. Mais especificamente, pretende-se apreender como as relações entre disciplinaridade e interdisciplinaridade incidem sobre as articulações entre artefato científico e propositividade política nas narrativas sobre sustentabilidade nos artigos observados.

Para tanto, o artigo discorre, primeiro, sobre a forma de seleção dos artigos analisados e explicita os norteamentos teórico-metodológicos da análise com ênfase nos fundamentos sociológicos que possibilitam o escrutínio sobre a potencialidade sociopolítica das propostas e a busca para um entendimento "equivalente" entre cientificidades diferentes. Apresenta, depois, a descrição dos arranjos conceituais que estruturam as diferentes as propostas de sustentabilidade existentes nos artigos para demonstrar o tipo de articulação conceitual predominante em cada conjunto, suas recorrências interdisciplinares, assim como a contribuição destas para a formulação diferentes tipos de propositividade. Por fim, desenvolve uma síntese sobre os sentidos de interdisciplinaridade encontrados, reconhecidos aqui pelos termos ecologização e politização, bem como a definição de um princípio comum entre eles, denominada como interdisciplinaridade por continuidade. O artigo termina com uma breve reflexão sobre as implicações desses resultados quanto às suas potencialidades e limites para o enfrentamento da problemática da sustentabilidade.

\section{Os contornos da pesquisa}

Foram selecionados quinze dos vinte e cinco artigos mais citados no sítio Web of Science, entre 1990 e 2010, em cada uma das áreas de ecologia, economia, sociologia e antropologia. Os critérios de pesquisa foram: (1) título: sustainab* (de forma a abranger sustainability e sustainable); (2) categoria: ecology, economics, sociology e anthropology; (3) tipo de documen- 
to: article. O resultado possibilitou uma amostra de artigos que não tem pretensão de ser representativa de toda produção das ciências aqui selecionadas sobre o tema, nem uma resposta geral ao problema da relação entre especialização e hibridismo. Mas garantiu um ponto de partida relevante para a análise sociológica direcionada à relação entre artefato científico e propositividade política, aqui denominada de abordagem socioepistêmica.

Quais seriam os contornos dessa abordagem? Abrigada sob o manto dos estudos sociais das ciências, a análise buscou apreender a dinâmica social e a potencialidade política expressas nos próprios artigos, em busca do agenciamento potencial dos enunciados científicos. Esse enfoque fundamenta-se naquilo que é comumente denominado problema da extensão (Collins; Evans, 2010), a partir do questionamento das relações entre o artefato já produzido e a maneira como este se apresenta ao debate sobre as estratégias de enfrentamento dos problemas junto a esferas mais amplas da dinâmica social contemporânea. Desse modo, não há a pretensão de se reproduzir a tendência, predominante nos science studies, de evidenciar o trabalho social subjacente ao conjunto de artigos analisados, como forma de se salientar a indissociabilidade entre as dinâmicas sociopolítica e epistemológica na produção dos artefatos científicos, próximo daquilo que é caracterizado como problema da legitimidade (Collins; Evans, 2010). Longe de se desprezar essa última perspectiva, a intenção é utilizá-la como ponto de partida seguro para a primeira, como forma de observar os processos sociais e políticos que podem derivar de tais artefatos.

Ou seja, o que possibilita a análise de diferentes perspectivas científicas sob o mesmo olhar e em suas derivações é a concepção de que não somente a produção científica está impregnada por processos socioculturais e políticos, mas, também, que as ciências se configuram como espaços privilegiados de produção de artefatos socioculturais e políticos - perspectiva adotada por autores com posturas teóricas diferenciadas no interior dos 
Sociologias, Porto Alegre, ano 16, no 37, set/dez 2014, p. 42-70

science studies, como Latour (1994), Knorr-Cetina (1999), Collins (1985), Yearley (2005) e Pickering (1992). Nesse sentido, cada ciência aqui representada é compreendida como cultura epistêmica, ou seja, [...]amalgams of arrangements and mechanisms - bonded through affinity, necessity, and historical coincidence - which, in a given field, make up how we know what we know (Knorr-Cetina, 1999, p. 1). No entanto, para evitar a ênfase disciplinar sugerida pela autora, entende-se aqui "cultura" como [...] conjunto de estruturações potenciais da experiência, capaz de suportar conteúdos tradicionais variados e de absorver novos (Viveiros de Castro, 2002, p. 209). Essa perspectiva possibilita conceber uma dada ciência pela forma particular de construção dos arranjos entre os seus conteúdos internos e elementos exógenos, sejam eles científicos ou não. A questão torna-se, então, compreender como cada cultura epistêmica alimenta suas experiências com a apreensão dos elementos exteriores à sua dinâmica.

Assim, cada ciência envolvida nesse estudo pode ser concebida como um campo amplo, permeável, dinâmico e sem fronteiras claramente definidas. Sob esse prisma, ao analisar as relações entre disciplinaridade e interdisciplinaridade, não se busca a apreensão da institucionalização científica, mas de perspectivas socioepistêmicas de concepção e ação na dinâmica do mundo contemporâneo. Essa posição explica a opção de observar as relações entre disciplinaridade e interdisciplinaridade em ecologia, economia, sociologia e antropologia e não nas ciências de "interface", tais como ecologia humana, ecologia política, economia ecológica, dentre outras. Estas, nessa abordagem, são compreendidas como tentativas de institucionalização da interface entre eventos humanos e não humanos como temática específica, sem, contudo, se desvincular das perspectivas oriundas das ciências antes mencionadas.

Para apreender as configurações possíveis do tema de forma equivalente sem desperdiçar a riqueza específica de cada abordagem, a leitura

http://dx.doi.org/10.1590/15174522-016003703 
dos artigos foi orientada para identificação e articulação de três dimensões presentes em narrativas científicas sobre sustentabilidade: (1) a concepção ontológica de interação entre eventos humanos e não humanos; (2) a noção de perturbação, que abrange ameaças, riscos e vulnerabilidades; e, por fim, (3) as estratégias de tratamento dos dilemas ambientais a que se dedicam. A primeira dimensão ancora-se em Yearley, para quem[...] the distinctive contribution of Science and Technology Studies research is to see that the very business of 'knowing nature' shapes the knowledge that results; this decisively influences how effective or not such knowledge is in other public contexts (Yearley, 2008, p. 923). O conhecer a natureza como campo de atenção analítica implica tanto na tentativa de reconhecimento da dinâmica das interações ecológicas, quanto na definição de fronteiras/correlações entre eventos humanos e não humanos como base para a construção de estratégias de enfrentamento dos problemas ambientais. Observar como essas fronteiras/correlações são concebidas em cada conjunto de artigos possibilitou um olhar para as tendências teórico-conceituais utilizadas em diferentes narrativas sustentabilidade.

A outras duas dimensões metodológicas decorrem das contribuições sobre a noção de risco (Beck, 2010) como um elemento que reconfigura os quadros da modernidade, de forma geral, e as relações entre ciência e política, de forma particular. Sob essa perspectiva, riscos, ameaças e perturbações - produzidos pela saturação dos usos tecnocientíficos - evidenciam a condição paradoxal da produção científica: ela é, ao mesmo tempo, produtora, instrumento de definição e possível fonte de solução dos riscos (Beck, 2010). São essas duas últimas atribuições que interessam ao presente estudo sobre sustentabilidade. Se a ciência tem papel relevante nos processos de construção das políticas de enfrentamento dos riscos ambientais, talvez seja interessante compreender como diferentes ciências abordam essas dimensões: a definição das ameaças e seu papel na formulação de diretrizes de ação. 
A junção das duas orientações para a formulação da tríade analítica tem como fundamento a ideia de que as condições de identificação das situações de ameaça e de definição de estratégias de tratamento só são possíveis no interior de um campo ontológico de distribuição dos diferentes eventos - humanos e não humanos - instalados nos diferentes arcabouços teóricos utilizados. Assim, ao analisar os artigos a partir do direcionamento desses três aspectos, foi possível identificar tanto os repertórios conceituais utilizados nos conjuntos de artigos analisados quanto as formas de articulação entre essas três dimensões. Foi possível, também, observar os sentidos de interdisciplinaridade que permeiam algumas das narrativas de sustentabilidade.

A "aplicação" da tríade metodológica nos artigos se constituiu por meio de uma leitura em certo sentido prospectiva, ao buscar identificar as três dimensões de análise na narrativa de cada artigo. Isso permitiu a compilação dos arranjos teórico-conceituais que configuram distintas visões de sustentabilidade. Possibilitou, também, analisar como esses arranjos engendram a propositividade expressa nos artigos. Além de uma visão bastante ampla de como perspectivas teóricas e conceituais se apresentam nas propostas analisadas, foi possível observar as lacunas e continuidades entre essas perspectivas no que se refere aos seus sentidos de interdisciplinaridade. Infelizmente, devido às dimensões do artigo, a análise detalhada dos textos não foi contemplada, privilegiando-se, assim, a síntese dos arranjos conceituais e suas derivações propositivas e interdisciplinares.

\section{Arranjos de sustentabilidade, interdisciplinaridade e propositividade}

Nos artigos em ecologia, as concepções de interação entre eventos humanos e não humanos apresentam-se intimamente relacionadas 
com as propostas e estratégias de solução. O arcabouço teórico-conceitual ecológico que concebe as interações configura-se como o campo de estruturação da propositividade da maioria dos artigos. As formas de entendimento sobre riscos e ameaças, contudo, são dotadas de grande generalidade e não se configuram como o locus analítico das narrativas. Disso estruturam-se dois tipos de arranjos conceituais, aqui denominados de manutenção da integridade ecológica e manutenção das funções ecológicas. A primeira abordagem é representada por Lubchenco et al. (1991), Kessler et al. (1992), Lindenmayer et al. (2000) e Richter et al. (2003). São propostas de recuperação/conservação da integridade ecológica, diretamente relacionadas à persistência, nos anos 1990, de uma concepção ontológica de dissociação entre "humanidade" e "natureza", imprimindo-se uma visão de competição ou de rivalidade entre as duas dimensões. O conceito de ecossistema apresenta-se como base para essas interações. Isso possibilita uma atenção especial tanto para perspectivas de conservação da biodiversidade por meio de gestão adaptativa tecnicista, ou seja, pela exaltação do conhecimento ecológico como guia dos esforços de ajustamento das técnicas e estratégias de planejamento frente à complexidade dos sistemas ecológicos.

À medida que esforços de conjunção entre eventos sociais e ecológicos vão se tornando mais internalizados, a noção de integridade perde espaço para as propostas direcionadas à manutenção das funções ecológicas. Esse arranjo, já presente em artigos das fases iniciais da análise (Holling, 1993; Costanza e Daly, 1992; Ugliati e Brown, 1998; van denBergh e Verbruggen, 1999; Ugliati e Brown, 1998; e Doran, 2002), encontra-se mais estruturado nos artigos de 2006 a 2010 (De Groot, 2006; Ness et al., 2007; Choi et al., 2008; Termorshuizen e Opdam, 2009; Chapin et al., 2010). A crescente preocupação com o tema mudanças ambientais globais, como um evento composto por dimensões sociais e ecológicas indissociáveis, gera um entendimento velado de que a integridade não 
se apresenta como um objetivo factível, tornando-se premente, portanto, a manutenção das funções ecológicas fundamentais. Esse deslocamento se apresenta em conjunto com o processo de descentramento do conceito de ecossistema, acompanhado agora pelo conceito de paisagem. O fortalecimento do manejo adaptativo orientado para uma perspectiva mais politizada e a incorporação das demandas sociais à problemática da sustentabilidade são características importantes desse arranjo.

No que se refere à interdisciplinaridade, a colaboração entre economia e ecologia se torna mais estreita conforme o deslocamento entre os dois arranjos descritos acima se efetiva. Na perspectiva da integridade, a colaboração é mais rarefeita, não apenas por ser uma expressão do efeito da dissociação ontológica que a acompanha, mas, sobretudo, porque uma perspectiva tecnoecológica de manejo adaptativo apresenta-se como suficiente para a sustentabilidade. A noção de valor ecológico intrínseco contribui para esse quadro, onde as formulações mais propriamente ecológicas dominam as narrativas. Por sua vez, o fortalecimento gradativo da ideia de manutenção das funções ecológicas afina-se com o processo de aproximação entre ecologia e economia. A estruturação das propostas a partir de um escopo interacional dos sistemas socioecológicos complexos, possibilita a emergência de concepções utilitaristas de valor dos elementos ecológicos, abrindo espaço para a constituição de uma ampla articulação com a teoria econômica. Assim, o reconhecimento de que é possível - diante das mudanças ambientais - constituir apenas um esforço de manutenção das funções ecológicas, pressupõe a tradução, em termos econômicos, da importância ou do valor dessas para a construção das estratégias de sustentabilidade. Como ciências que derivam de uma mesma raiz", esse "reencontro" econômico-ecológico se traduz,

\footnotetext{
${ }^{1}$ É bem reconhecida a influência de Thomas Malthus, um dos pais fundadores da economia moderna, na estruturação do pensamento darwiniano com o seu Ensaio sobre a População (Malthus, 1983), bem como a definição de ecologia de Ernest Haeckel como a "economia da natureza" (Foster, 2005).
} 
nos artigos ecológicos, nos binômios "ecossistemas/capital natural" e "funções/serviços ecológicos". Nas perspectivas em que a base conceitual é o conceito de paisagem, o conceito de capital natural não é utilizado, mas mantém-se a relação direta entre "função" e "serviço".

A tentativa de operacionalização desse diálogo no âmbito político se constitui pela premissa do adaptive management. Num primeiro momento, surge um entendimento mais técnico do termo, sendo encarado como "ajustamento" ou "resposta" aos processos de experimentação e monitoramento ecológico sobre os ecossistemas. Nos últimos anos, o termo se torna mais politizado, característica que pode ser expressa pela substituição do termo management por stewardship (Chapin et al., 2010). Esta mudança se realiza com a justificativa de que o último seria uma expressão mais adequada para o tipo de estratégia necessária à formulação de políticas de sustentabilidade em contextos de rápida mudança ambiental e de grande incerteza, por prever uma maior inserção dos agentes nas estratégias de redução das condições de vulnerabilidade. Em ambos, persiste a noção de que as dimensões sociais e ecológicas dos sistemas têm em comum a adaptive capacity.

O conjunto de artigos econômicos apresenta - tal como os ecológicos - uma consistente articulação entre as concepções de interação entre eventos humanos e não humanos e as propostas de solução. Riscos e ameaças, apesar da caracterização econômica, mantêm um alto nível de generalidade e abstração. Isso expressa tanto certa ausência de centralidade analítica aos problemas ambientais quanto a interpretação de que esses são uma espécie de consenso estruturante do debate, um "fato" incontestável que dispensa o uso de grande energia analítica. Talvez seja pela ênfase nas interações que os primeiros artigos busquem uma demarcação crítica entre as tendências "ambiental" e "ecológica", sendo o instrumental teórico-metodológico dessa última considerado como mais adequado do que a 
primeira para o entendimento das questões de sustentabilidade (Howarth e Norgaard, 1992; Asheim, 1994; Stern et al., 1996 e Binswanger, 2001).

Disso, delineiam-se dois arranjos conceituais: sustentabilidade como manutenção do capital natural e como construção político-econômica. Na primeira, a base fundamental é a noção de complexidade sistêmica, na qual eventos humanos e não humanos interagem por meio de fluxos materiais e equalizam-se como diferentes "capitais", instâncias nas quais seus dinamismos produzem "renda" ou "produtividade" apropriável. É justamente nesse fluxo que residem as propostas desse primeiro arranjo, próprio dos artigos publicados na virada dos anos 1990 e 2000 (Toman, 1994; Hinterberger et al., 1997; Costanza et al., 1998; Farber et al., 2002 e Ekins et al., 2003). A noção de escala ou controle de fluxo domina as perspectivas de regulação sustentável dos processos intertemporais de alocação. Mesmo artigos críticos à noção de capital natural, prevendo certa distância entre sistemas ecológicos e sistemas econômicos (Hinterberger et al., 1997), a ideia de regulação dos fluxos permanece. Não obstante, a dimensão propositiva desse arranjo tende ao gerenciamento cientificista, com ênfase às noções de "monitoramento", "avaliação" e "mensuração".

No segundo arranjo, a construção político-econômica de sustentabilidade, o conceito de capital natural perde espaço para a construção de cenários de sustentabilidade. A noção de complexidade sistêmica mantém-se como configuração ontológica fundamental, mas a preocupação com a contabilidade dos fluxos é substituída por estratégias de planejamento territorial politicamente costurado entre agentes econômicos e políticos. Nesses artigos (Kay et al., 1999; Rotmans, 2000 e Ciegis et al., 2009), inclusive os que não se fundamentam no conceito de cenário (Robinson, 2004; Reed et al., 2006 e Martinez-Alier et al., 2010), o direcionamento das estratégias de sustentabilidade como ato proeminentemente político ganha força, deixando as formulações tecnocientíficas em segundo plano. 
Sobre os exercícios de interdisciplinaridade, nos primeiros anos, a abordagem crítica da perspectiva ecológica sobre a ambiental se faz no campo econômico, já que as narrativas buscam deslegitimar a pertinência propriamente econômica das últimas. Ou seja, o debate de legitimação da perspectiva econômico-ecológica se estrutura mais pela "inconsistência" teórica neoclássica quanto ao dilema da sustentabilidade do que pela necessidade de se evidenciar o contorno ecológico desprezado por ela. Portanto, é nos artigos adeptos da manutenção do capital natural que o diálogo com a ecologia é inevitável. Em um modelo de colaboração próximo ao que se apresenta nos artigos ecológicos, a caracterização econômica dos eventos ecológicos se generaliza, mas, contudo, o arcabouço ecológico promove um espraiamento integrativo sobre os temas econômicos. Não há espaço aqui para a ideia de valor "intrínseco" dos elementos ecológicos. A lógica utilitária de mensuração dos serviços ecossistêmicos é dominante.

Porém, na perspectiva da construção político-econômica de sustentabilidade, os processos colaborativos deslocam-se gradativamente do prisma ecológico (Kay et al., 1999; Rotmans, 2000 e Ciegis et al., 2009) para o sociológico (Robinson, 2004 e Martinez-Alier et al., 2010), próximo daquilo que é comumente denominado de ecologia política. No prisma ecológico, os processos políticos estão internalizados nos sistemas complexos e, portanto, são aspectos importantes da capacidade adaptativa a esses sistemas em diferentes dimensões/escalas. No prisma sociológico, buscam-se, geralmente, formas de articulação entre abordagem econômico-ecológica e tendências sociológicas construcionistas (Robinson, 2004) ou pelo resgate do ecologismo radical proveniente dos anos 1960 no que se refere a crítica à noção de crescimento inerente aos processos de modernização (Martinez-Alieret al., 2010). Se no prisma ecológico a integração entre dimensões ecológicas, econômicas e sociopolíticas se articula via capacidade adaptativa, no sociológico parece persistir um 
relativo distanciamento entre a dimensão "científica", provedora de informações válidas, e a dimensão "sociopolítica", exposta ao jogo dos valores e interesses. O que justifica colocar os dois prismas no mesmo arranjo é a forma pela qual os artefatos científicos, tanto ecológicos quanto econômicos, se apresentam não como os elementos definidores de políticas mas como repertórios que informam processos de interlocução política, tanto no sentido macro quanto no micro.

No conjunto sociológico, a articulação entre concepções de interação e formulação de estratégias também prevalece nas narrativas, mas de uma forma diferente daquela apresentada nos conjuntos ecológicos e econômicos. Se, nesses últimos, é possível vislumbrar certa autonomia entre "interações" e "ameaças", essa distinção não é claramente detectável no sociológico. Distante das concepções universalizantes que se manifestam nos termos "humanidade" ou "ação humana", a prerrogativa sociológica de se compreenderem as dimensões sociais no contexto da modernidade faz com que as interações sejam concebidas como eminentemente problemáticas. No entanto, é possível perceber que, nos artigos analisados, o olhar sociológico não se dirige empiricamente em direção aos problemas ambientais.

Isso contribui com a formulação de três arranjos conceituais: as perspectivas contributiva, socioecológica e sociopolítica de sustentabilidade. Na primeira, a explicação sociológica mantém-se distanciada de uma formulação própria sobre sustentabilidade (Gale e Cordray, 1994; Saltiel et al., 1994; Hassanein e Kloppenburg, 1995; Meares, 1997; Peter et al., 2000; McKenzie-Mohr, 2000 e van den Berg et al., 2007). Esses estudos focam práticas que acompanham soluções técnicas sustentáveis, como forma de se dimensionar as condições sociais e psicológicas de disseminação e êxito, gerando, assim, uma propositividade secundária que pode ou não fortalecer processos já em curso e formulados além e aquém da esfera sociológica.

Uma possível aproximação da explicação sociológica ao tema resulta no arranjo aqui denominado de perspectiva socioecológica. Nessa, 
ocorre uma incorporação de tendências ecológico-econômicas (Olson, 1995, Hunter, 1997; Briassoulis, 2002) ou ecológico-geográficas (Sneddon et al. 2002) - fundamentadas nas teorias de sistemas complexos - à perspectiva contributiva. Emerge daí a tendência de se ressaltar a importância de temas sociais ao arcabouço construído pelas ciências da natureza. O turismo e os conflitos sociais em torno de recursos hídricos são trabalhados como aspectos que devem ser reconhecidos como constituintes dos sistemas socioecológicos complexos.

Essa abordagem tem como contraponto uma perspectiva sociopolítica que, por sua vez, tem representantes desde 1997 (Michalos, 1997), mas se torna bem estabelecida entre 2001 e 2010 (Plugliese, 2001; Spaargaren, 2003 e Mol, 2007). Nesses artigos, o uso da teoria social contemporânea como fundamentação torna-se recorrente, enfatizando-se a articulação entre diferentes dimensões como forma de se refletir sobre a potencialidade política das propostas de sustentabilidade. Assim, a articulação entre dimensões sociotécnicas e sociopolíticas (Plugliese, 2001; Mol, 2007), modos de vida e estruturas sociais (Spaargaren, 2003) e os dilemas entre complexidade fenomenológica e especialização científica (Michalos, 1997) impulsionam a formulação das estratégias de sustentabilidade.

Obviamente, essa reorientação possibilita a passagem de uma propositividade contributiva para o desenvolvimento de uma agenda propriamente sociológica sobre as questões de sustentabilidade. No entanto, essa dinâmica distancia as narrativas sociológicas dos processos de colaboração com as ciências naturais e econômicas, já que apenas em um artigo (Pugliese, 2001) busca-se o entendimento sociológico sobre dimensões não humanas, via teoria ator-rede (Callon, 1986; Latour, 2000). Os demais artigos não se dedicam a um tratamento mais atento aos aspectos ecológicos, configurando-se, assim, em uma abordagem de tendência sociocêntrica. A ênfase na teoria social tende a tomar a problemática da sustentabilidade a partir do trânsito geográfico (a mobilidade e os fluxos 
entre esferas locais e globais) e dimensional (entre agência e estrutura social ou entre práticas sociais e instituições) como base para a formulação de políticas ambientais. Assim, o caminho para uma propositividade mais encorpada em termos sociológicos configura-se num processo de interiorização ao seu próprio arcabouço teórico-conceitual.

Nos artigos antropológicos, por sua vez, apresenta-se uma articulação diferente, se comparada aos demais conjuntos de artigos. Se nesses, cada um ao seu modo, prevalece uma estreita relação entre concepções de interação e propostas de solução, no conjunto antropológico o fio condutor das narrativas é a definição e a descrição das situações de ameaça. Aqui, o caráter etnográfico dos estudos antropológicos é direcionado para situações ambientalmente problemáticas. Isso não significa que concepções de interações sejam pouco importantes para o desenvolvimento das propostas, como veremos. No entanto, os diferentes prismas pelos quais essas inserções etnográficas se orientam possibilitam arranjos conceituais que oferecem resultados bastante diferentes no que se refere à formulação propositiva.

O primeiro desses arranjos pode ser denominado de ecológico-cultural. Os artigos dessa perspectiva caracterizam-se por circunscrever a problemática da sustentabilidade, de forma geral e não automática, à questão da capacidade de suporte(Clay e Lewis, 1990; Humphries, 1993; Goldman, 1995; Henrich, 1997; Mccabe, 2003; Davies e Wismer, 2007). Isso significa que o conceito não é explicitamente citado, mas está presente sua lógica de articulação entre eventos humanos e não humanos pela perspectiva da escassez. Isso leva, também, ao entendimento de que a maioria dos artigos rejeita a concepção neomalthusiana do conceito, mas a considera como referência a ser problematizada. Com o foco nas situações de transformação sociocultural e produtiva entre os campos tradicional/moderno, local/global, subsistência/mercado, dentre outros, essas narrativas problematizam a relação entre qualidade (enquanto ajustamento social e ecológico permiti- 
do por condições de vida etnicamente demarcadas) e quantidade (enquanto extensão demográfica e produtiva) diante de processos de modernização e exposição às forças de mercado. Essa relação "quali/quanti" tende a se configurar em condições de não sustentabilidade à medida que a exposição às forças exógenas potencializa o quantitativo, colocando em xeque o qualitativo. Esses processos são normalmente contextualizados em abordagens socioecológicas, não apenas pela relação de escassez, mas também pela via dos sistemas complexos. E são focalizados em contextos geralmente não urbanos e em condições comunitárias.

Além desse arranjo ecológico-cultural, emerge dos artigos outro arranjo facilmente denominável de perspectiva sociocultural. Nesse, apresentam-se tentativas tanto de evidenciar situações etnicamente demarcadas, também em processo de transição em campos antagônicos (Dove e Kammen,1997; Narcher e Hickey, 2002; Fratkin e Mearns, 2003; Agrawal, 2003; Blaser, 2009), quanto de extensão da análise antropológica para contextos "modernos" ou urbano-industriais (Wikan, 1995; Kloppenburget al., 2000; Cone e Myhre, 2000; Murphy, 2004). No primeiro caso, dirige-se a atenção para a importância das dimensões "subjetivas" que influenciam as situações de mudança socioambiental, assim como para o dinamismo interno de grupos sociais frente à formulação exógena das políticas ambientais que os atingem. No segundo, analisam-se etnograficamente práticas de sustentabilidade ou eventos problemáticos com relação às mudanças ambientais globais. De forma geral, procura-se demonstrar, em contraponto à ideia de ajustamento, as condições de diversificação de respostas e enfrentamentos possibilitadas por perspectivas socioculturais diferenciadas. Nesse sentido, proliferam-se estudos comparativos sobre o posicionamento de grupos sociais "tradicionais" e "modernos" a uma mesma situação de ameaça ou ao choque de perspectivas entre esses diante de uma mesma configuração territorial. 
Do ponto de vista de suas articulações interdisciplinares, os dois arranjos seguem, obviamente, caminhos distintos. No arranjo ecológico-cultural encontra-se uma articulação bem estabelecida entre o arcabouço ecológico e descrição etnográfica. Apesar de oferecer leituras empíricas detalhadas sobre as configurações socioecológicas a que se dedica, a ênfase no entendimento dos processos de ajustamento tende a apresentar resultados pré-estabelecidos de análise. Ou seja, a presença do arcabouço socioecológico tende a restringir a diversidade interpretativa dos grupos ou situações analisadas aos mecanismos de adaptabilidade dos eventos humanos aos eventos não humanos. Apesar disso, a aceitação da postura etnográfica incorporada ao escopo ecológico se converte em uma propositividade particularizada, fortemente atrelada ao contexto analisado, com tentativas de intervenção direta nas políticas ambientais locais.

Por outro lado, a perspectiva sociocultural se desloca de uma postura etnográfica tipicamente "antropológica" quanto às sociedades "não ocidentais", para o interesse em temas relacionados aos agenciamentos sociotécnicos, aos riscos tecnocientíficos e aos processos sociais reflexivos. Isso demonstra uma aproximação ao universo temático historicamente delimitado como "sociológico". Apresentando uma diversidade maior de referências teórico-conceituais, essa perspectiva inclina-se a duas orientações propositivas: a tentativa de evidenciar os aspectos intangíveis e a dinâmica interna das sociabilidades analisadas, como forma de "culturalizar" os processos de definição de estratégias de sustentabilidade; a segunda volta-se à própria teoria socioantropológica no esforço de se construir uma agenda própria sobre diversidade cultural e questões ambientais.

Assim, nesse conjunto antropológico, o tipo de propositividade predominante é resultado da articulação entre a descrição etnográfica das situações de ameaça e concepção de interação entre diferentes eventos. Se esses são compreendidos pela perspectiva ecológico-cultural, a propositividade tende 
Sociologias, Porto Alegre, ano 16, no 37, set/dez 2014, p. 42-70

à recomendação "particularista", atenta aos processos de redefinição das políticas ambientais localizadas no contexto problematizado. Se a perspectiva é a do ordenamento sociocultural, a propositividade se dirige ao debate sobre a pertinência conceitual das ciências sociais diante da complexidade das questões socioambientais como passo anterior a ser dado para, num segundo momento, dirigir-se a intervenção nas políticas ambientais.

\section{Os sentidos de interdisciplinaridade}

Duas conclusões fundamentais derivam da análise desses arranjos conceituais. A primeira refere-se à disposição conceitual com relação à orientação metodológica da pesquisa. Existe uma forte tendência de articulação entre concepções de interação e estratégias de enfrentamento, no sentido de que as primeiras estruturam o campo de possibilidades das segundas. Essa articulação se apresenta em três dos quatro conjuntos (ecológico, econômico e sociológico), tendo como exceção os artigos antropológicos que, em maioria, tendem a privilegiar as situações de risco e ameaça como fio condutor das narrativas.

A articulação dominante (concepções de interação - proposta de solução) apresenta uma tendência mais propositiva se comparada com os artigos antropológicos. Em contrapartida, esses arranjos tendem a uma menor propensão de diálogo com perspectivas exógenas à concepção estabelecida internamente. Faz sentido, portanto, que, nesses esquemas, as situações de ameaça sejam concebidas "por alto". Imprescindíveis para a estruturação de um mundo que necessita de estratégias de solução, as crises, ameaças e riscos estão sempre presentes nos artigos analisados, mas, em muitos casos, estas estão no plano das certezas que, de tão certas, não necessitam de um tratamento central nesse tipo de narrativa. 
Por outro lado, a iniciativa antropológica de construção de narrativas apresenta uma relação consolidada entre a análise das situações de ameaça e o caráter etnográfico. Isso não significa que a formulação teórica acerca das interações não desempenhe papel relevante no desenvolvimento das estratégias de enfrentamento cunhadas pelos artigos antropológicos. Obviamente, a maneira como essas ameaças são caracterizadas está intimamente relacionada à preocupação antropológica de conceber a particularidade de interações que compõem cada sociabilidade por ela estudada. No entanto, junto a essa configuração, emerge uma flexibilidade de diálogo entre arranjos conceituais - nesse caso, entre perspectivas ecológico-cultural e sociocultural - com relação às possíveis contribuições de um para o outro na qualificação dos problemas tratados. Duas possíveis explicações: primeiro, o olhar etnográfico pode ser considerado, de certo modo, autônomo em relação às perspectivas teóricas apresentadas, o que permite certos deslocamentos entre perspectivas; segundo, as situações de ameaça, terreno privilegiado da incerteza, possibilitam interstícios de entendimento onde a sobreposição de conceitos oriundos de distintas tradições pode existir.

A segunda conclusão refere-se aos sentidos teórico-conceituais de interdisciplinaridade derivados dos arranjos. É possível afirmar a existências de dois campos de articulação não excludentes entre si e que, portanto, se interpenetram: ecologização e politização. Por ecologização compreende-se um tipo de exercício interdisciplinar a partir da convergência entre narrativas ajustadas ao estilo ecológico de entendimento das interações. Esse estilo conta com um arcabouço teórico-metodológico bem delimitado, grande uniformidade conceitual e um suporte semântico claro no que se refere à interligação entre eventos humanos e não humanos. Todas as iniciativas interdisciplinares construídas sob essa égide, presentes em todos os conjuntos, se caracterizam pela aceitação tácita 
dessa estrutura e pela redefinição de elementos conceituais exógenos em conformidade a ela. Portanto, a interdisciplinaridade por ecologização não acontece de forma simétrica, mas a partir do transbordamento de suas prerrogativas sobre os elementos que compõem a estrutura epistêmica exógena, configurando-se em um procedimento metonímico ou epistemofágico de incorporação. Dela emerge uma forma de propositividade que tende a formular estratégias que melhor ajustam a ação humana aos princípios gerais de reprodução do que é cientificamente concebido como dinâmica ecológica, levando em consideração o crescente entendimento desses humanos como partícipes dessa configuração.

Por politização compreende-se outro modelo de fazer interdisciplinar, no qual o entendimento sobre a mobilização dos coletivos humanos - geralmente considerados como "complexos", mas não necessariamente "sistêmicos" - deve ser preponderante na formulação das estratégias de sustentabilidade. Nessa seara, as articulações entre perspectivas científicas devem ser levadas em conta para a construção de ambientes favoráveis para a "melhor" tomada de decisão. No campo da politização, contudo, não existe a preponderância de um arcabouço teórico-conceitual, como na ecologização. A proliferação de construções teóricas é marca fundamental desse campo. De forma geral, paira uma concepção de que a ação humana é dotada de maior desprendimento em relação aos elementos ecológicos ou uma necessidade maior de consideração dos eventos humanos nos processos ecológicos, a partir de um entendimento desses eventos para além da concepção de "ajustamento" ecológico. No limite, tende-se a ver os processos de politização nos próprios elementos ecológicos, seguindo uma linha aberta de forma radical por Latour (2004), mas que encontra uma recente versão mais ponderada em Ingold (2012).

A interdisciplinaridade por politização se constitui por afinidade temática, onde conceitos e teorias são colocados em colaboração como 
estratégia de qualificação da análise. Apesar da preponderância sociológica sobre esse modelo, a ausência de um arcabouço teórico hegemônico não possibilita um processo de colaboração metonímico, tal como se apresenta na ecologização. A politização implica num modelo de propositividade em que as diversas perspectivas científicas devem olhar reflexivamente para si mesmas inseridas nesse ambiente politizado, como forma de garantir o fornecimento de informação qualificada à definição de estratégias.

Assim, é possível observar um progressivo deslocamento da ecologização para a politização como campo preponderante de exercício interdisciplinar, sendo, ao mesmo tempo, o primeiro bem estabelecido, enquanto que o segundo apresenta-se em processo de estruturação. No entanto, em cada um dos conjuntos de artigos e em seus arranjos conceituais, a colaboração interdisciplinar se inscreve conforme se apresenta a dosagem entre as duas searas discursivas.

Colocando-se ecologização e politização como polos antagônicos que se espraiam como campos discursivos, mas tendentes à sobreposição, quanto mais próximo está o conjunto de artigos de um desses polos de irradiação, maior é a capacidade de formulação de sentidos de interdisciplinaridade. Ou seja, são esses os conjuntos de artigos que representam cientificidades que impulsionam o sentido de articulação, que vai da disciplinaridade para a interdisciplinaridade. Esses polos são representados pelos artigos ecológicos e sociológicos. Não é a esmo, portanto, que esses dois conjuntos são os que fornecem arranjos conceituais dotados, cada um ao seu modo, de certo grau de autonomia discursiva. Isso não significa que eles estejam totalmente imunes à influência do centro de irradiação oposto ao seu sentido. Os conjuntos de artigos dispostos mais ao centro desses campos de irradiação - econômicos e antropológicos - estão mais expostos aos sentidos de interdisciplinaridade. Os artigos econômicos estão mais próximos da ecologização, porém, apresentam-se crescente- 
mente influenciados pelos processos de politização. Os artigos antropológicos estariam mais próximos da politização, mas, do mesmo modo, estão bastante expostos aos processos de ecologização.

Se, como vimos, a ecologização converge para um arcabouço teórico-metodológico compartilhado e a politização converge para temáticas compartilhadas, é possível afirmar que, nos artigos analisados, o sentido de interdisciplinaridade relacionado à problemática da sustentabilidade se constitui pela tendência de afastamento dos estranhamentos discursivos, temáticos e teórico-metodológicos. Isso se efetiva a partir de processos em que a "colaboração" por afinidade supera a tendência de "confronto" entre perspectivas discrepantes. O caráter de continuidade operacionaliza um modelo de articulação às interações entre humanos e não humanos, sem abrir mão, contudo, de certa homogeneidade de apreensão empírica, formulação conceitual e destilação teórico-metodológica. Promove-se, assim, um processo interdisciplinar sem romper a dinâmica disciplinar que as constituem, o que aponta para uma espécie de interdisciplinaridade por continuidade. De forma geral, ela pode ser considerada como um esforço eficaz de formulação dos arranjos conceituais que caracterizam as concepções de sustentabilidade, impulsionado pelo reconhecimento de que disciplinaridade e especialização científica não são suficientes para a resolução dos dilemas ambientais. Isso viabiliza conexões, mas dificulta a inserção de elementos conceituais não previstos nesses campos de continuidade semântica. Ou seja, a interdisciplinaridade por continuidade converge paradoxalmente para aquilo que pretende ultrapassar, ao estimular o surgimento de novas disciplinaridades, como é possível observar em Kates et al. (2001), cujo objetivo é construir uma ciência da sustentabilidade a partir dos fundamentos próprios dos sistemas socioecológicos complexos.

Talvez esse paradoxo persista, porque boa parte todos os esforços realizados para a superação da dicotomia "sociedade"|“natureza" - seja 
da perspectiva da ecologização ou da politização - tenha lançado mão de procedimentos metonímicos de extensão das propriedades de uma seara a outra, como pode ser observado na utilização do sentido ecológico do termo "adaptação" aos eventos humanos ou no uso da noção socioantropológica de "agenciamento" aos eventos não humanos. Não que esses procedimentos sejam inválidos, destituídos de capacidade analítica ou de potencialidade política. Mas, por operarem pela lógica da continuidade, eles impedem a colaboração pautada pela descontinuidade entre perspectivas. Na bibliografia analisada, os arranjos conceituais sob a égide da ecologização - principalmente nos conjuntos ecológicos e econômicos - apresentam forte demanda de inclusão de questões "sociais", sem, contudo, se configurar um diálogo com arcabouços socioantropológicos. Essa condição aponta para a incipiência de tratamento conjunto entre abordagens díspares que não resultem na redução de uma à outra. Se há alguma abertura para interdisciplinaridade entre perspectivas não continuadas ou discrepantes, esta se apresenta, timidamente, nos artigos que abrem espaço para a centralidade nas situações de risco e ameaça.

Quais seriam, então, as condições para o surgimento de uma interdisciplinaridade por estranhamento, descontinuidade ou interstícios? Se, por uma interpretação tipicamente sociológica, a "interdisciplinaridade por continuidade" pode ser expressa em termos de uma "afinidade eletiva" (Weber, 2004), quais seriam os esforços necessários para a emergência de uma interdisciplinaridade guiada por "discrepâncias eletivas"?

Roberto Donato da Silva Junior. Sociólogo, Doutor em Ambiente e Sociedade pelo Programa de Pós-Graduação em Ambiente e Sociedade do Núcleo de Estudos e Pesquisas Ambientais (NEPAM) do Instituto de Filosofia e Ciências Humanas da Universidade Estadual de Campinas (UNICAMP). Professor do Centro de Ciências Humanas e Sociais Aplicadas da Pontifícia Universidade Católica de Campinas (PUC-CAMPINAS). Desenvolveu pesquisa sobre a relação entre organizações não-governamentais e populações locais junto às propostas de etnoconservação, com apoio da Coordenação de Aperfeiçoamento de Pessoal de Nível Superior 
(CAPES) e pesquisa sobre a intersecção epistemológica entre ecologia, sociologia e antropologia nas propostas de sustentabilidade. $\lambda$ robertodnt@yahoo.com.br

Leila da Costa Ferreira. Doutora em Ciências Sociais pela Universidade Estadual de Campinas. Pós doutoramento na Universidade do Texas / EUA em Políticas Públicas e Ambiente, e Pós Doutoramento na Universidade de York / Inglaterra em Teoria Social e Ambiente. Professora Titular da Universidade Estadual de Campinas. Coordenadora do Grupo de Estudos Brasil/ China junto ao Centro de Estudos Avançados da UNICAMP. $\longrightarrow$ leilacf@unicamp.br

Thomas Michael Lewinsohn. Biólogo, Mestre e Doutor em Ecologia pela UNICAMP. Professor Titular do Departamento de Biologia Animal no Instituto de Biologia da UNICAMP. Coordenou o Programa de Pós-Graduação em Ecologia da Unicamp. Foi Pesquisador ou Professor Visitante na Univ. Bayreuth, Alemanha (1989); no NERC Centre for Population Biology, Imperial College, Inglaterra; NCEAS - National Center for Ecological Analysis and Synthesis, USA; Estación de Doñana, Espanha; Univ. Nac. Córdoba, Argentina (2005). Editor de área da revista Biotropica. $\longrightarrow$ thomasl@unicamp.br

\section{Referências}

1. AGRAWAL, A. Sustainable governance of common-pool resources: Context, methods, and politics. Annual Review of Anthropology, n. 32, p. 243-262, 2003.

2. ASHEIM, G. B. Net national product as an indicator of sustainability. Scandinavian Journal of Economics, v. 96, n. 2, p. 257-265, 1994.

3. BECK, U. Sociedade de Risco: rumo a uma outra modernidade.São Paulo: Editora 34, 2010.

4. BINSWANGER, M. Technological progress and sustainable development: what about the rebound effect? Ecological Economics, v. 36, n. 1, p. 119-132, jan. 2001.

5. BLASER, M. The Threat of the Yrmo: The Political Ontology of a Sustainable Hunting Program. American Anthropologist, v. 111, n. 1, p. 10-20, 2009.

6. BRIASSOULIS, H. Sustainable tourism and the question of the commons. Annals of Tourism Research, v. 29, n. 4, p. 1065-1085, 2002.

7. CALLON, M. Elementspour une sociologie de la traduction:La domestication des coquilles Saint-Jacques et des marinspecheurs en baie de Saint-Brieuc, L ‘Anee sociologique,vol. 36, 1986, p. 169-208. 
8. CHAPIN, F. S. et al. Ecosystem stewardship: sustainability strategies for a rapidly changing planet. Trends in Ecology \& Evolution, v. 25, n. 4, p. 241-249, Apr. 2010.

9. $\mathrm{CHOI}, \mathrm{Y}$. D. et al. Ecological restoration for future sustainability in a changing environment. Ecoscience, v. 15, n. 1, p. 53-64, 2008.

10. CIEGIS, R.; RAMANAUSKIENE, J.; MARTINKUS, B.The Concept of Sustainable Development and its Use for Sustainability Scenarios. Inzinerine Ekonomika-Engineering Economics, n. 2, p. 28-37, 2009.

11. CLAY D. C.; LEWIS L. A. Land-use, soil loss, and sustainable agriculture in Rwanda. Human Ecology, v. 18, n. 2, p. 147-161, 1990.

12. COLLINS, H. M., Changing Order: Replication and Induction in Scientific Practice, Beverley Hills \& London: Sage, 1985.

13. COLLINS, H. M.; EVANS, R. Repensando a expertise. Belo Horizonte: Fabrefactum, 2010.

14. CONE, C. A.; MYHRE, A. Community-supported agriculture: A sustainable alternative to industrial agriculture? Human Organization, v. 59, n. 2, p. 187-197, 2000.

15. COSTANZA, R. et al. Principles for sustainable governance of the oceans. Science, v. 281, n. 5374, p. 198-199, jul. 1998.

16. COSTANZA, R.; DALY, H. E. Natural capital and sustainable development. Conservation Biology, v. 6, n. 1, p. 37-46, mar. 1992.

17. DAVIES, E. G. R.; WISMER, S. K. Sustainable forestry and local people: The case of Hainan's Li minority. Human Ecology, v. 35, n. 4, p. 415-426, 2007.

18. DE GROOT, R. Function-analysis and valuation as a tool to assess land use conflicts in planning for sustainable, multi-functional landscapes. Landscape and Urban Planning, v. 75, n. 3-4, p. 175-186, mar. 2006.

19. DORAN, J. W. Soil health and global sustainability: translating science into practice. Agriculture Ecosystems \& Environment, v. 88, n. 2, p. 119-127, Feb. 2002.

20. DOVE, M. R.; KAMMEN, D. M. The epistemology of sustainable resource use: Managing forest products, swiddens, and high-yielding variety crops. Human Organization, v. 56, n. 1, p. 91-101, 1997.

21. EKINS, P. et al. A framework for the practical application of the concepts of critical natural capital and strong sustainability. Ecological Economics, v. 44, n. 2-3, p. 165-185, mar. 2003.

22. FARBER, S. C.; COSTANZA, R.; WILSON, M. A. Economic and ecological concepts for valuing ecosystem services. Ecological Economics, v. 41, n. 3, p. 375-392, jun. 2002. 
23. FERREIRA, LEILA. C.; VIOLA, E. (Orgs.). Incertezas de sustentabilidade na globalização.Campinas: Editora da Unicamp, 1996.

24. FRATKIN, E.; MEARNS, R. Sustainability and pastoral livelihoods: Lessons from East African Maasai and Mongolia. Human Organization, v. 62, n. 2 p. 112-122, 2003.

25. GALE, R, P.; CORDRAY, S. M. Making sense of sustainability - 9 answers to what should be sustained. Rural Sociology, v. 59, n. 2, p. 311-332, 1994.

26. GOLDMAN, A. Threats to Sustainability in African Agriculture - Searching For Appropriate Paradigms. Human Ecology, v. 23, n. 3 p. 291-334, 1995.

27. HASSANEIN, N.; KLOPPENBURG, J. R. Where the grass grows again: Knowledge exchange in the sustainable agriculture movement. Rural Sociology, v. 60 n. 4, p. 721-740, 1995.

28. HENRICH, J. Market incorporation, agricultural change, and sustainability among the Machigueuga Indians of the Peruvian Amazon. Human Ecology, v. 25, n. 2, p. 319-351, 1997.

29. HINTERBERGER, F.; LUKS, F.; SCHMIDTBLEEK, F. Material flows vs. natural capital - What makes an economy sustainable? Ecological Economics, v. 23, n. 1, p. 1-14, Oct. 1997.

30. HOLLING, C. S. Investing in research for sustainability. Ecological Applications, v. 3, n. 4, p. 552-555, Nov. 1993.

31. HOWARTH, R. B.; NORGAARD, R. B. Environmental valuation under sustainable development. American Economic Review, v. 82, n. 2, p. 473-477, May 1992.

32. HUMPHRIES, S. The intensification of traditional agriculture among yucatecmaya farmers - facing up to the dilemma of livelihood sustainability. Human Ecology, v. 21, n. 1 , p. $87-102,1993$.

33. HUNTER, C. Sustainable tourism as an adaptive paradigm. Annals of Tourism Research, v. 24, n. 4, p. 850-867, 1997.

34. INGOLD, T. Trazendo as coisas de volta à vida: emaranhados criativos num mundo de materiais. Horizontes Antropológicos, Porto Alegre, v. 18, n. 37, jun.2012 .

35. KATES, R.W. et al. Sustainability science. Science, v. 292, p. 641-642, 2001.

36. KAY, J. J. et al. An ecosystem approach for sustainability: addressing the challenge of complexity. Futures, v. 31, n. 7, p. 721-742, Sep. 1999.

37. KESSLER, W. B. et al. New perspectives for sustainable natural-resources management.Ecological Applications, v. 2, n. 3, p. 221-225, Aug. 1992. 
38. KLOPPENBURG, J.; LEZBERG, S.; DE MASTER, K. et al. Tasting food tasting sustainability: Defining the attributes of an alternative food system with competent, ordinary people. Human Organization, v. 59, n. 2, p. 177-186, 2000.

39. KNORR-CETINA, K. Epistemic Cultures: How the Sciences Make Knowledge. Cambridge, MA: Harvard University Press. 1999.

40. LATOUR, B. Ciência em ação: como seguir cientistas e engenheiros sociedade afora. São Paulo: UNESP, 2000.

41. LATOUR, B. Jamais Fomos Modernos. Ensaio de Antropologia Simétrica. Rio de Janeiro: Editora 34, 1994.

42. LATOUR, B. Políticas da natureza: como fazer ciência na democracia. Bauru: EDUSC, 2004.

43. LINDENMAYER, D. B.; MARGULES, C. R.; BOTKIN, D. B. Indicators of biodiversity for ecologically sustainable forest management. Conservation Biology, v. 14, n. 4, p. 941-950, Aug. 2000.

44. LUBCHENCO, J.et al. The Sustainable Biosphere Initiative: An Ecological Research Agenda. Ecology,72(2): 371-412, 1991.

45. MARTINEZ-ALIER, J. et al. Sustainable de-growth: Mapping the context, criticisms and future prospects of an emergent paradigm. Ecological Economics, v. 69, n. 9, p. 1741-1747, Jul. 2010.

46. MCCABE, J. T. Sustainability and livelihood diversification among the Maasai of northern Tanzania. Human Organization, v. 62, n. 2, p. 100-111, 2003.

47. MCKENZIE-MOHR, D. Promoting sustainable behavior: An introduction to community-based social marketing. Journal of Social Issues, v. 56, n. 3, p. 543$554,2000$.

48. MEARES, A. C. Making the transition from conventional to sustainable agriculture: Gender, social movement participation, and quality of life on the family farm. Rural Sociology, v. 62, n. 1, p. 21-47, 1997.

49. MICHALOS, A. C. Combining social, economic and environmental indicators to measure sustainable human well-being. Social Indicators Research, v. 1, n.2, p. 221-258, 1997.

50. MITCHELL, G.; MAY, A.; MCDONALD, A. PICABUE - A methodological framework for the development of indicators of sustainable development. International Journal of Sustainable Development and World Ecology, v. 2, n. 2, p. 104-123, Jun. 1995.

51. MOL, A. P. J. Boundless biofuels? Between environmental sustainability and vulnerability. Sociologia Ruralis, v. 47, n. 4, p. 297-315, 2007. 
52. MURPHY, R. Disaster or sustainability: The dance of human agents with nature's actants. Canadian Review of Sociology and Anthropology, v.41, n. 3, p. 249-266, 2004.

53. NATCHER, D. C. HICKEY, C. G. Putting the community back into communitybased resource management: A criteria and indicators approach to sustainability. Human Organization, v. 61, n. 4, p, 350-363, 2002.

54. NESS, B. et al. Categorising tools for sustainability assessment. Ecological Economics, v. 60, n. 3, p. 498-508, Jan. 2007.

55. OLSON, R. L. Sustainability as a social vision. Journal of Social Issues, v. 51, n. 4, p. 15-35, 1995.

56. PETER, G.et al. Coming back across the fence: Masculinity and the transition to sustainable agriculture. Rural Sociology, v. 65, n. 2, p. 215-233, 2000.

57. PICKERING, A. Science as practice and culture. Chicago; London: University of Chicago, 1992.

58. PUGLIESE, P. Organic farming and sustainable rural development: A multifaceted and promising convergence. Sociologia Ruralis, v. 41, n. 1, p. 112-130, 2001.

59. REED, M. S.; FRASER, E. D. G.; DOUGILL, A. J. An adaptive learning process for developing and applying sustainability indicators with local communities. Ecological Economics, v. 59, n. 4, p. 406-418, Oct. 2006.

60. RICHTER, B. D.; MATHEWS, R.; WIGINGTON, R. Ecologically sustainable water management: Managing river flows for ecological integrity. Ecological Applications, v. 13, n. 1, p. 206-224, Feb. 2003.

61. ROBINSON, J. Squaring the circle? Some thoughts on the idea of sustainable development. Ecological Economics, v. 48, n. 4, p. 369-384, Apr. 2004.

62. ROTMANS, J. et al. Visions for a sustainable Europe. Futures, v. 32, n. 9-10, p. 809-831, Nov-Dec 2000.

63. SALTIEL, J.; BAUDER, J. W.; PALAKOVICH, S. Adoption of sustainable agricultural practices - diffusion, farm structure, and profitability. Rural Sociology, v. 59, n. 2, p. 333-349, 1994.

64. SNEDDON, C.; HARRIS, L.; DIMITROV, R. et al. Contested waters: Conflict, scale, and sustainability in aquatic socioecological systems. Society \& Natural Resources, v. 15, n.8, p. 663-675, 2002.

65. SPAARGAREN, G. Sustainable consumption: A theoretical and environmental policy perspective. Society \& Natural Resources, v. 16, n .8, p. 687-701, 2003. 
66. STERN, D. I.; COMMON, M. S.; BARBIER, E. B. Economic growth and environmental degradation: The environmental kuznets curve and sustainable development. World Development, v. 24, n. 7, p. 1151-1160, Jul 1996.

67. TERMORSHUIZEN, J. W.; OPDAM, P. Landscape services as a bridge between landscape ecology and sustainable development. Landscape Ecology, v. 24, n. 8, p. 1037-1052, Oct 2009.

68. TOMAN, M. A. Economics and sustainability - balancing trade-offs and imperatives. Land Economics, v. 70, n. 4, p. 399-413, Nov. 1994.

69. ULGIATI, S.; BROWN, M. T. Monitoring patterns of sustainability in natural and man-made ecosystems. Ecological Modelling, v. 108, n. 1-3, p. 23-36, May 1998.

70. VAN DEN BERG, A. E.; TERRY, H. HENK, S. Preference for nature in urbanized societies: Stress, restoration, and the pursuit of sustainability. Journal of Social Issues, v. 63, n. 1, p. 79-96, 2007.

71. VAN DEN BERGH, J.; VERBRUGGEN, H. Spatial sustainability, trade and indicators: an evaluation of the 'ecological footprint'. Ecological Economics, v. 29, n. 1, p. 61-72, Apr. 1999.

72. VIVEIROS DE CASTRO, E. A inconstância da alma selvagem e outros ensaios de antropologia. São Paulo: Cosac \& Naify, 2002.

73. WEBER, M. A ética protestante e o espírito do capitalismo. São Paulo: Companhia das Letras, 2004.

74. WIKAN, U. Sustainable Development In The Mega-City - Can The Concept Be Made Applicable. Current Anthropology, v. 36, n. 4, p. 635-655, 1995.

75. YEARLEY, S. Making Sense of Science: Understanding the Social Study of Science. London; Thousand Oaks; New Delhi: Sage Publications, 2005.

76. YEARLEY, S. Nature and the environment in science and technology studies. In: HACKETT, E. J. et al.(Eds.). The Handbook of Science and Technology Studies. Cambridge: MIT Press, 2008.

Recebido em: 29/01/2014

Aceite final: 02/07/2014 